\title{
First XMM-Newton observations of a cataclysmic variable I: Timing studies of OY Car ${ }^{\star}$
}

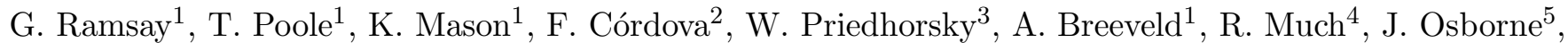

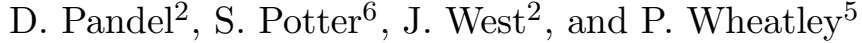 \\ 1 Mullard Space Science Lab., University College London, Holmbury St. Mary, Dorking, Surrey, RH5 6NT, UK \\ 2 Department of Physics, University of California, Santa Barbara, CA 93106, USA \\ 3 Los Alamos National Laboratory, Los Alamos, NM 87545, USA \\ 4 Astrophysics Division, ESTEC, 2200, AG Noordwijk, The Netherlands \\ 5 Department of Physics \& Astronomy, University of Leicester, University Road, Leicester, LE1 7RH, UK \\ ${ }^{6}$ South African Astronomical Observatory, PO Box 9, Observatory 7935, South Africa
}

Received 27 September 2000 / Accepted 17 October 2000

\begin{abstract}
We present XMM-Newton observations of the eclipsing, disc accreting, cataclysmic variable OY Car which were obtained as part of the performance verification phase of the mission. The star was observed 4 days after an outburst and then again 5 weeks later when it was in a quiescent state. There is a quasi-stable modulation of the X-rays at $\sim 2240 \mathrm{~s}$, which is most prominent at the lowest energies. We speculate that this may be related to the spin period of the white dwarf. The duration of the eclipse ingress and egress in X-rays is $20-30 \mathrm{~s}$. This indicates that the bulk of the X-ray emission originates from the boundary layer which has a negligible height above the surface of the white dwarf. The eclipse profile implies a white dwarf of mass $M_{1}=0.9-1.1 M_{\odot}$ and a secondary star of $M_{2}=0.08-0.11 M_{\odot}$.
\end{abstract}

Key words. accretion, accretion discs - binaries: eclipsing - stars: individual: OY Car - novae, cataclysmic variables - X-rays: stars

\section{Introduction}

Cataclysmic variables $(\mathrm{CVs})$ are close binary systems in which the secondary (usually a dwarf main sequence star) fills its Roche lobe and transfers material onto the white dwarf primary. In non-magnetic systems, this material forms an accretion disc around the primary. Some of these show "dwarf nova" outbursts when the system brightens by several magnitudes for a few days or weeks. These outbursts recur on a timescale of weeks to months.

High inclination systems provide an opportunity to locate the X-ray emitting region(s). Eclipses are not seen in the X-ray emission of OY Car (Naylor et al. 1988) nor in the EUV (Mauche \& Raymond 2000) during outbursts, suggesting that the prime X-ray source (the boundary layer, where the disc is decelerated and merges with the white dwarf) is obscured at all phases. The residual X-ray flux is thought to be due to scattering from an accretion disc wind. In contrast, during quiescence X-ray eclipses

Send offprint requests to: G. Ramsay,

e-mail: gtbr@mssl.ucl.ac.uk

* Based on observations obtained with XMM-Newton, an ESA science mission with instruments and contributions directly funded by ESA Member States and the USA (NASA). have been seen in HT Cas (Wood et al. 1995), Z Cha (van Teeseling 1997) and OY Car (Pratt et al. 1999) when the boundary layer is not hidden by disc material. The duration of the eclipse ingress/egress shows that the X-ray source is small and close to the photosphere of the white dwarf, $<1.15 R_{\mathrm{wd}}$ in HT Cas (Mukai et al. 1997).

$\mathrm{X}$-ray observations of dwarf novae have been hampered by their relatively low flux levels. Using instruments with large effective areas, such as XMM-Newton (Jansen et al. 2001), we can examine the eclipse profile of these systems with unprecedented precision and accurately locate the source of the X-ray flux. Having an orbital period of $1.51 \mathrm{~h}$, OY Car was an ideal target for the performance verification phase of XMM-Newton. Specifically, OY Car was chosen to test the timing capability of the $X M M$ Newton Optical Monitor (OM; Mason et al. 2001). This paper presents an initial analysis of the optical and X-ray light curves. In a companion paper (Ramsay et al. 2001), we present the results of an analysis of the X-ray spectra.

\section{Observations}

OY Car was observed using XMM-Newton on 29-30 June 2000 and again on 7 Aug. 2000. Optical observations from 
Table 1. The duration of the EPIC observations and the mean background subtracted count rates

\begin{tabular}{lrrr}
\hline Date & PN & MOS1 & MOS2 \\
\hline $29-30$ June 2000 & $48 \mathrm{ksec}$ & $51 \mathrm{ksec}$ & $51 \mathrm{ksec}$ \\
orbit 102 & $1.09 \mathrm{ct} / \mathrm{s}$ & $0.43 \mathrm{ct} / \mathrm{s}$ & $0.41 \mathrm{ct} / \mathrm{s}$ \\
7 Aug. 2000 & $7 \mathrm{ksec}$ & $14 \mathrm{ksec}$ & $14 \mathrm{ksec}$ \\
orbit 121 & $0.56 \mathrm{ct} / \mathrm{s}$ & $0.17 \mathrm{ct} / \mathrm{s}$ & $0.19 \mathrm{ct} / \mathrm{s}$ \\
\hline
\end{tabular}

the AAVSO (Mattei 2000a) indicate that OY Car was in outburst on $24-25$ June (reaching $~ 12 \mathrm{mag}$ ) and had faded below $\sim 15$ mag by 27 June. Observations taken using the OM indicate that $V \sim 16.0-16.2 \mathrm{mag}$ on 29 June, indicating that it had faded rapidly after outburst. On 7 Aug., OM observations gave $B \sim 15.7-15.9$ mag. A series of 5 optical spectra were also taken at the South African Astronomical Observatory on 7 Aug. The spectra imply $V \sim 15.8-16.5$ and $B \sim 15.6-16.8$, indicating that, in the optical, OY Car was at a similar brightness compared to the June observation.

Outbursts of OY Car vary in length. For those that last less than a week, AAVSO observations (Mattei 2000b) show that the next outburst typically occurs 180 days later, so it is unlikely that OY Car experienced another outburst between the two XMM-Newton observations.

Data were collected in various observation modes of the OM. These include $V$ band images and fast window mode data in UVW1 $(\sim 2400-3400 \AA)$ (all taken in June) and $B$ band images $(\sim 3800-4900 \AA)$ and also Grism data (all taken in August). Although OY Car was faint in both the UV and optical Grism observations, an emission line is detected at $\mathrm{H} \alpha$. Observations were also made with each EPIC camera (PN, MOS1 \& MOS2, 0.1-12 keV; Turner et al. 2001). There was a weak detection $\left(0.05 \mathrm{ct} \mathrm{s}^{-1}\right.$ background subtracted) of OY Car in the RGS $(0.3-2.1 \mathrm{keV}$; den Herder et al. 2001).

The EPIC exposures were taken in full window mode using the medium filter. In both EPIC observations, the particle background increased significantly towards the end. The data with high background were therefore removed from the analysis. The resulting useful exposure and the mean background subtracted count rates are shown in Table 1: in X-rays OY Car faded by a factor of 2.1 between the two observations.

In the June observations, 9 eclipses were observed in the EPIC cameras and 2 in the OM UVW1 filter in fast mode. In the August observations, 2 and 3 eclipses were observed in the EPIC PN and MOS cameras respectively and 3 eclipses in the OM $B$ filter in fast window mode. For the EPIC data we concentrate on the June observations since the source was brighter and more eclipses were observed. The data were processed using the XMM-Newton Science Analysis System released on 12 July 2000.

\section{Light curves}

\subsection{Extracting the light curves}

Light curves were extracted from all 3 EPIC cameras using apertures $\sim 30^{\prime \prime}$ in radius centered on OY Car, chosen so that the aperture did not cover more than one CCD. This radius encompasses $\sim 90$ percent of the integrated PSF (Aschenbach et al. 2000). Background lightcurves were extracted from the same CCD on which the source was detected, scaled and subtracted from the source light curves.

To increase the signal to noise ratio, the counts from the three EPIC cameras were summed, having first corrected for absolute timing discrepancies by crosscorrelation and alignment of the sharp eclipse ingress and egress. Similarly we extracted background subtracted light curves from the OM fast mode data. All our light curves were then phased on the orbital period and aligned in such a manner that phase $\phi \sim 0.0$ was centered on the eclipse.

\subsection{General features}

Figure 1 shows the EPIC light curve, split into $0.1-1.0 \mathrm{keV}$ and $1.0-4.0 \mathrm{keV}$ energy bands. We also plot the ratio of counts in the $0.1-1.0 \mathrm{keV}$ and $1.0-4.0 \mathrm{keV}$ bands. There is very little variation in the $1.0-4.0 \mathrm{keV}$ band apart from the deep eclipses. In the soft band, however, there are significant quasi-sinusodial variations, with corresponding variations in spectra shape. These are not fixed in orbital phase.

\subsection{Non-orbital modulations}

To explore the variations in the light curve further, we constructed a Discrete Fourier Transform of the summed EPIC light curve. We show the amplitude spectrum in the energy range $0.1-12.0 \mathrm{keV}$ in the top panel of Fig. 2 . This amplitude spectrum is contaminated by harmonics of the orbital period. To subtract this effect, we removed the eclipses from the light curve. The resulting amplitude spectra $(0.1-12.0 \mathrm{keV}, 0.1-1.0 \mathrm{keV}$ and $1.0-4.0 \mathrm{keV})$ are shown in the middle and lower panels of Fig. 2.

The strongest amplitude peak occurs at $2240 \pm 60 \mathrm{~s}$ (where the error is a quarter of the full width of the amplitude peak at half maximum), and this is most prominent at the lowest energies. The orbital sidebands of the $2240 \mathrm{~s}$ period are also present. However, there are several other peaks which have similar amplitude particularly at low frequencies. We also searched for much shorter periods (less than $100 \mathrm{~s}$ ) and found no significant amplitude peaks. As the OM fast mode data was short in duration it was not possible to make a detailed search for periods on a timescale of a few $1000 \mathrm{~s}$ in these data.

The data folded on the $2240 \mathrm{~s}$ period are shown in Fig. 3. This confirms the energy dependance of the modulation, with an amplitude (maximum-minimum/the mean) of $\sim 43$ percent in the $0.1-1.0 \mathrm{keV}$ band and $\sim 20$ percent in the $1.0-4.0 \mathrm{keV}$ band. There is no evidence 

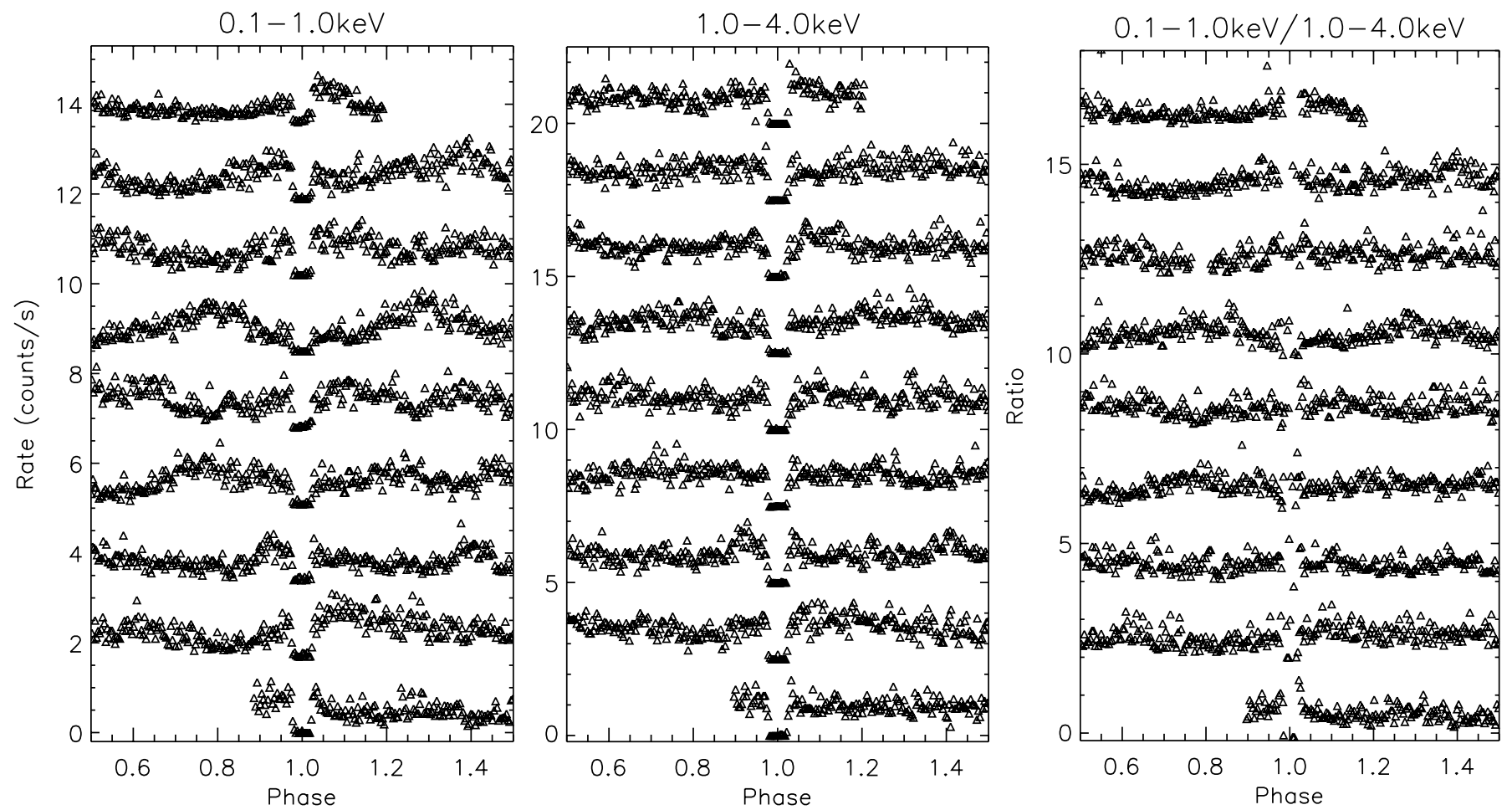

Fig. 1. The light curves obtained using the sum of data from all $3 \mathrm{EPIC}$ cameras in the $0.1-1.0 \mathrm{keV}$ (left) and $1.0-4.0 \mathrm{keV}$ (center) energy ranges and their ratio $0.1-1.0 \mathrm{keV} / 1.0-4.0 \mathrm{keV}$ (right hand panel) folded on the binary orbital period. The light curves have been binned into $10 \mathrm{~s}$ bins and offset vertically by $1.7 \mathrm{ct} / \mathrm{s}, 2.5 \mathrm{ct} / \mathrm{s}$ and 2.0 left to right respectively. Time increases from the bottom to the top

of a significant modulation in the $4.0-10.0 \mathrm{keV}$ band. In Fig. 4 we show the $0.1-1.0 \mathrm{keV}$ light curve phased on the $2240 \mathrm{~s}$ modulation. It is clear that if there is an underlying modulation its amplitude is variable. However, all the broad maxima appear centered on the phase range $\phi_{2240} \sim$ 0.6-1.1. We discuss the possible origin of the $2240 \mathrm{~s}$ period in Sect. 4.

\subsection{Multi-wavelength data}

We show in Fig. 5 simultaneous $X M M-N e w t o n$ X-ray/UV data which were obtained on 29-30 June 2000. These simultaneous data cover 2 eclipses (eclipses 6 and $7-\mathrm{cf}$. Fig. 1). We also show in Fig. 5 the $B$ filter $(3800-4900 \AA)$ fast mode data taken in 7 August. Apart from the eclipses, there is no variabilty in the UV data which corresponds to the low energy variations seen in soft X-rays. For instance, the dip which is seen in X-rays at $\phi \sim 1.4-1.5$ is more prominent in the $0.1-1.0 \mathrm{keV}$ energy band than at higher energies. However, the dip is not seen in the UV data. One orbital cycle later, there is a dip in soft X-rays between $\phi \sim 2.2-2.4$. These dips maybe related to the absorption features in the light curves of low mass X-ray binaries and in the dwarf nova U Gem (Mason et al. 1988). This phenomenon is thought to be due to the accretion disc having a significant vertical extent at certain azimuths.

\subsection{The eclipse profile}

In the optical the eclipse profile of disc accreting CVs is made up of several components (e.g. Wood et al. 1989). The eclipse of the white dwarf is defined to be centered on $\phi=0.0$ and has a sharp ingress and egress. The eclipse of the accretion disc is also symmetrical, but the ingress and egress are much more gradual. On the other hand the eclipse of the bright spot (where the accretion stream impacts with the accretion disc) is centered later in the orbital cycle. In the far-UV the white dwarf is the strongest component (Horne et al. 1994).

In Fig. 6 we show the mean eclipse profile in X-rays (0.1-1.0 keV and 1.0-4.0 keV), the near UV (2400-3400 $\AA$ ) and in $B(3800-4900 \AA)$. The immediate point to note is the very rapid ingress and egress in X-rays: 20-30 s, which is roughly the expected time to eclipse the diameter of the white dwarf. This suggests that practically all the $\mathrm{X}$-ray emission originates from the white dwarf. Unlike magnetic $\mathrm{CVs}$, which have relatively small accretion regions and have ingress times $<10 \mathrm{~s}$ (e.g. Steiman-Cameron \& Imamura 1999), the X-ray emission is not concentrated. Rather, the likely source is the boundary layer which is extended in azimuth around the white dwarf. There is also some (rather weak) evidence that the $1.0-4.0 \mathrm{keV}$ flux is eclipsed sooner and comes out of eclipse later than the 0.1-1.0 keV flux.

Also of note is the short duration of the eclipse: defining the eclipse duration as from first to fourth contact, we 


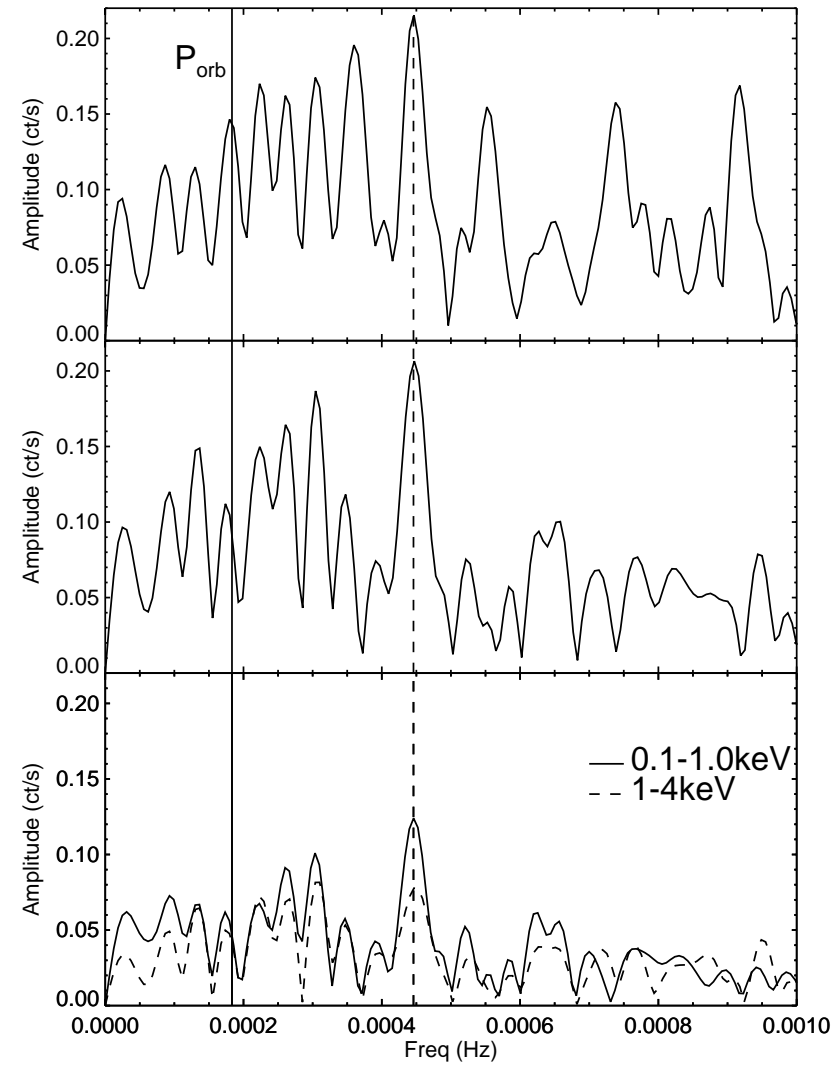

Fig. 2. Top panel: the amplitude spectrum of the background subtracted summed EPIC light curve (0.1-12.0 keV), Middle panel: the amplitude spectrum with the removal of the average eclipse profile $(0.1-12 \mathrm{keV})$, Bottom panel: as the middle panel but for energy ranges $0.1-1.0 \mathrm{keV}$ and $1.0-4.0 \mathrm{keV}$. The frequency of the orbital period corresponds to $P_{\text {orb }}$, while the dashed line corresponds to a period of $2240 \mathrm{~s}$

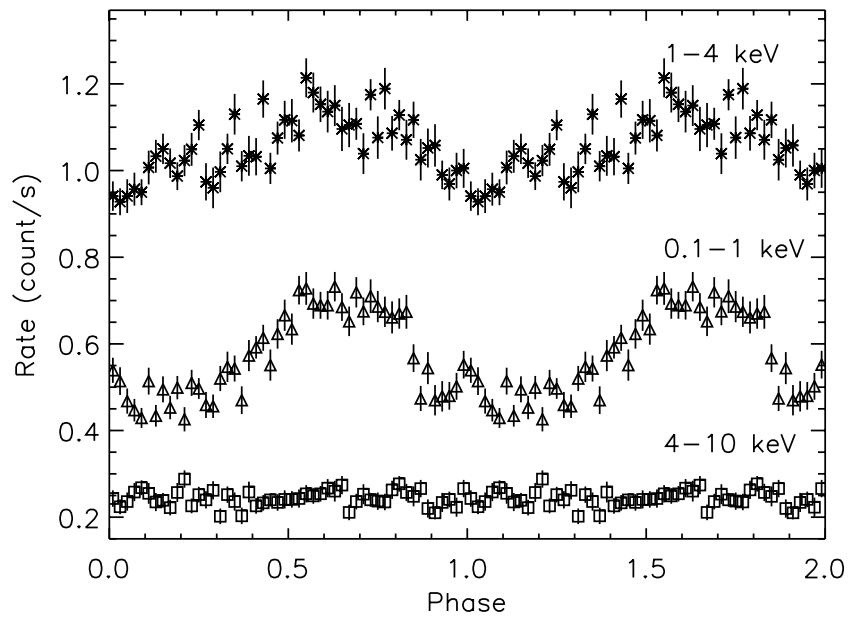

Fig. 3. The summed EPIC data with the eclipse profile removed and folded on the 2240 s period found from our Discrete Fourier Transform

find the duration is $290 \pm 10 \mathrm{~s}$ in X-rays, $278 \mathrm{~s} \pm 10 \mathrm{~s}$ in UVW1 and $286 \mathrm{~s} \pm 10 \mathrm{~s}$ in $B$. We discuss the implications of these results in Sect. 4. In the UVW1 filter the steep ingress/egress into/from eclipse starts at the same orbital phase as in X-rays. However, the flux does not return to

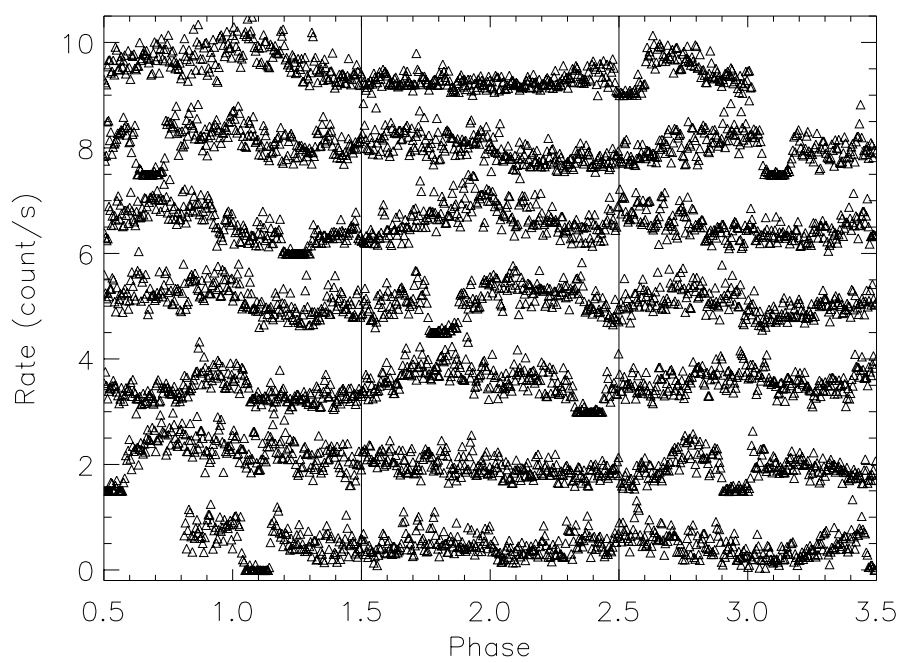

Fig. 4. The summed EPIC data $(0.1-1.0 \mathrm{keV})$ phased on the $2240 \mathrm{~s}$ period. Time increases from left to right and every 3 cycles the data have been offset vertically by $1.5 \mathrm{ct} / \mathrm{s}$
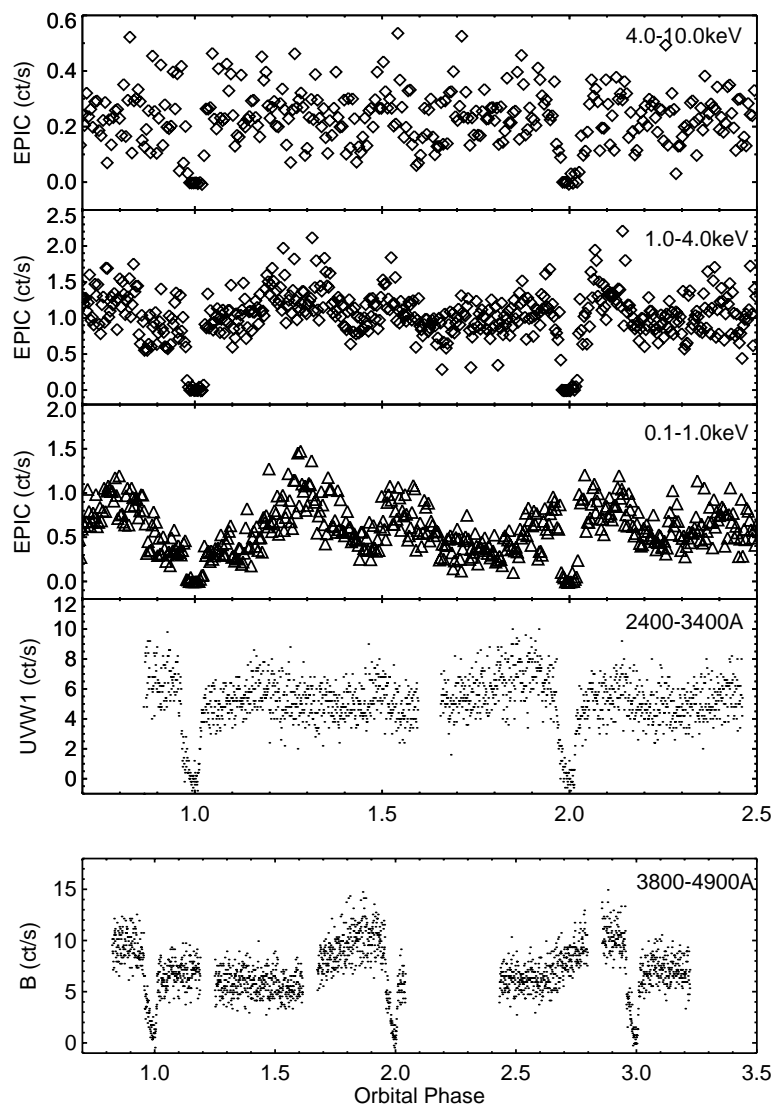

Fig. 5. Simultaneous EPIC (using the sum of the PN and MOS data) in the $0.1-1.0$ and $1.0-4.0 \mathrm{keV}$ energy bands and $\mathrm{OM}$ (UVW1 2400-3400 $\AA$ ) light curves taken on 29 June 2000. The OM ( $B$ filter) data were taken on 7 Aug. 2000. The OM data are binned into $5 \mathrm{~s}$ bins and EPIC data into $20 \mathrm{~s}$ bins apart from the $4-10 \mathrm{keV}$ data which is $30 \mathrm{~s}$ bins. Because of some uncertainty in their absolute time, the light curves were phased on the orbital period then aligned so that the eclipse was centered on $\phi=0.0$ 
its pre-eclipse flux - this is due to the fact that the bright spot (which is visible in the UVW1 band) is progressively foreshortened. In the $B$ filter (which was taken 5 weeks after the X-ray and UVW1 data), there is marginal evidence that the steep ingress starts before the ingress in X-rays. As in the UVW1 filter the flux does not return to its pre-eclipse flux.

\subsection{The mid-eclipse flux}

An image made using EPIC data taken from times when the source was eclipsed (defined as after 2nd contact and before 3rd) shows a faint source at the location of OY Car. Using all three EPIC detectors, we find a summed background subtracted count rate of $0.006 \pm 0.004 \mathrm{ct}^{-1}$ in the $1.0-12 \mathrm{keV}$ band and $0.027 \pm 0.004 \mathrm{ct}^{-1}$ in the $0.1-$ $1.0 \mathrm{keV}$ band. This suggests that there was some residual low energy X-ray flux during the eclipse.

To investigate this in greater detail we examined the source and background light curves of each eclipse in the three separate EPIC detectors. In eclipse 2, we found a

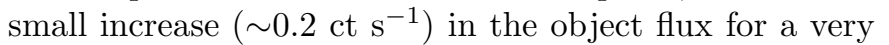
short time $(\sim 30 \mathrm{~s})$ in the EPIC PN which was not seen in the MOS detectors. We also found the object flux in eclipse 9 in the MOS 2 detector showed an increase towards the end of the eclipse which was not seen in either of the other detectors. We conclude that both these events were instrumental in origin. Excluding these flux enhancements which we do not regard as intrinsic to OY Car, we re-determined the background subtracted summed count rate during the eclipse: $0.003 \pm 0.003 \mathrm{ct}^{-1}(1.0-12.0 \mathrm{keV})$ and $0.024 \pm 0.004 \mathrm{ct}^{-1}(0.1-1.0 \mathrm{keV})$. The count rate in the $1.0-12.0 \mathrm{keV}$ energy band is not significant, but the $0.1-1.0 \mathrm{keV}$ count rate is significant at the $6 \sigma$ level.

There are several possible origins of this emission. One is the secondary star. Randich et al. (1996) found that $L_{\mathrm{X}} / L_{\text {bol }}$ saturates at $\sim 10^{-3}$ in late type stars, where $L_{\mathrm{X}}$ is the luminosity in the $0.1-2.0 \mathrm{keV}$ energy band. For a dwarf secondary star of $M_{2}=0.1 M_{\odot}$ (Sect. 4.2), and using the mass-luminosity relationship of Malkov et al. (1997), we find $\log \left(L_{\mathrm{bol}} / L_{\odot}\right)=-2.96$. This implies that $L_{\mathrm{X}}<410^{27} \mathrm{ergs} \mathrm{s}^{-1}$. To make a crude estimate of the luminosity of OY Car during the eclipse, we determine the ratio of the in-eclipse EPIC summed count rate over the mean EPIC summed count rate (for $0.1-1.0 \mathrm{keV}$ $\sim 0.045)$. We then scale the luminosity in the $0.1-1.0 \mathrm{keV}$ energy band (2 $10^{30}$ ergs s$^{-1}$, Ramsay et al. 2001) using this ratio and derive $\sim 910^{28} \mathrm{ergs} \mathrm{s}^{-1}$ for the in-eclipse luminosity assuming a distance of $82 \mathrm{pc}$ (Wood et al. 1989). Of course, the secondary star will have a softer spectrum than the integrated spectrum of OY Car, so our derived luminosity may be overestimated by a factor of $\sim 2$.

Even taking this into account, we find that the observed luminosity exceeds the maximum luminosity expected for a $0.1 M_{\odot}$ secondary by an order of magnitude. In order to match the estimated luminosity during eclipse, a secondary of mass $0.3 M_{\odot}$ would be required

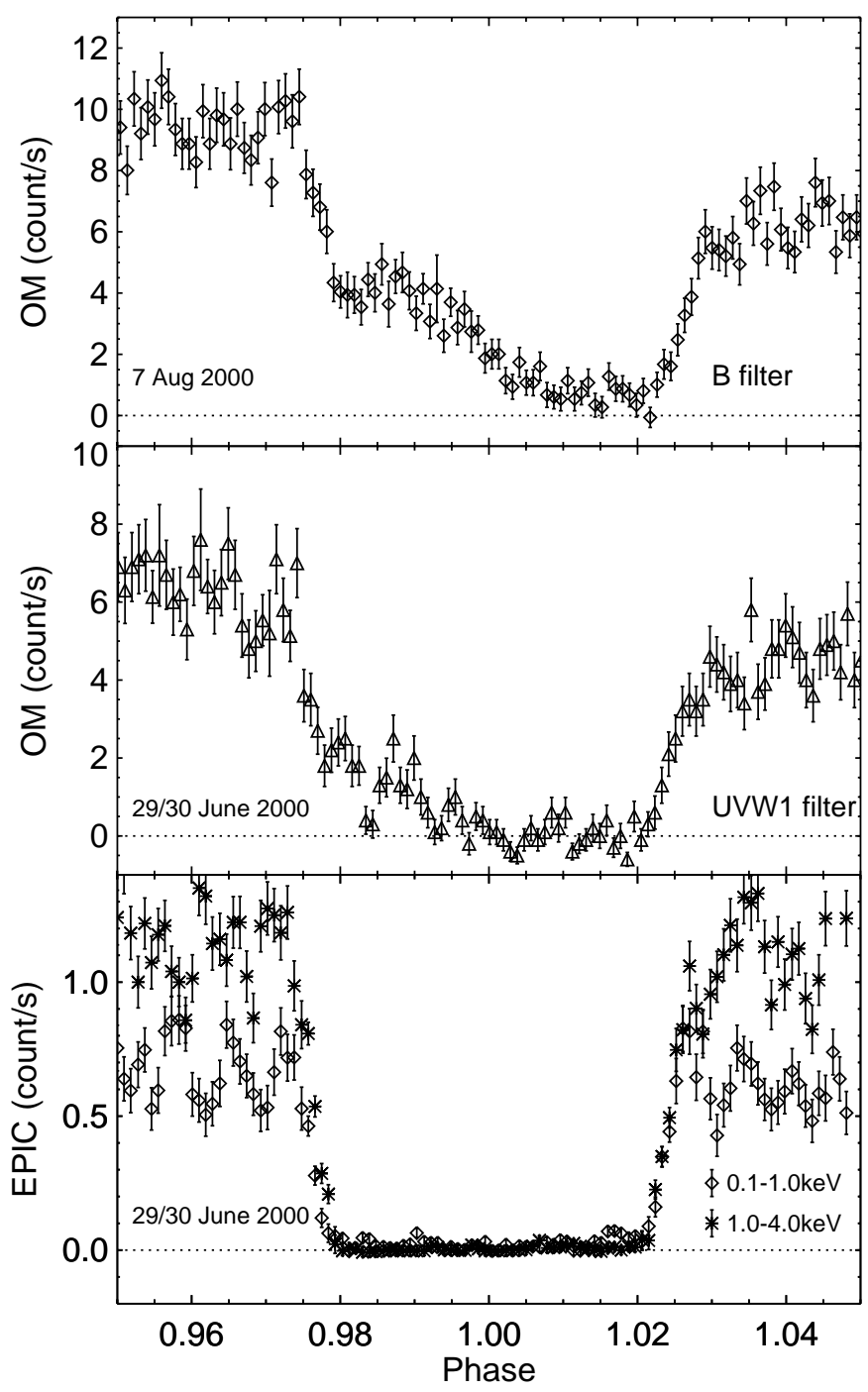

Fig. 6. Top panel: the eclipse profile in the OM $B$ filter $(3800-$ $4900 \AA$ ). Middle panel: as above but the OM UVW1 filter (2400-3400 A). Bottom panel: the eclipse profile obtained using the sum of data from all 3 EPIC cameras in the $0.1-1.0 \mathrm{keV}$ and 1.0-4.0 keV energy ranges. The data have been binned into $5 \mathrm{~s}$ bins

(Malkov et al. 1997). Such a mass is ruled out from our eclipse profile modelling (Sect. 4.2). We conclude that the weak detection of OY Car during eclipse is unlikely to be due to the secondary star. An alternative scenario, is the weak remnant of a large corona which is seen more prominently during outburst (e.g. Naylor et al. 1988). This seems to be more likely as an outburst was observed 4 days before the XMM-Newton observations.

\section{Discussion}

\subsection{The nature of the $2240 \mathrm{~s}$ period}

The X-ray light curve of OY Car exhibits quasi-sinusoidal variability that may have an underlying periodicity of $\sim 2240$ s. The variation is most prominent at softer energies but has a variable amplitude. Such a modulation 
could be due to energy dependent absorption, perhaps in disk material circulating above the orbital plane, or a quasi-stable bright spot on the photosphere of the white dwarf.

The latter scenario would imply that the spin period of the white dwarf $\left(P_{\text {spin }}\right)$ is $\sim 2240 \mathrm{~s}$. Marsh \& Horne (1998) observed OY Car in the UV towards the end of a superoutburst and found evidence for dwarf nova oscillations (17.94 and $18.15 \mathrm{~s}$ ). One suggestion was that one of these periods represented the Keplerian frequency of the inner edge of the accretion disc and the other a beat frequency between the Keplerian frequency and $P_{\text {spin }}$. This scenario implies $P_{\text {spin }} \sim 1500 \mathrm{~s}$. Interestingly, a period of $1500 \mathrm{~s}$ is close to the 1590 -second orbital sideband of the $2240 \mathrm{~s}$ period that we find in the amplitude spectrum (Fig. 2).

At this stage, we must regard this interpretation with a considerable degree of caution. However, it is interesting to note that a spin period of $P_{\text {spin }}=2240 \mathrm{~s}$, implies $P_{\text {spin }} / P_{\text {orb }}=0.41$. The two Intermediate Polars (weakly magnetic $\mathrm{CVs}$ ) which have orbital periods below the period gap - like OY Car - (RX J1238-38 and EX Hya) have $P_{\text {spin }} / P_{\text {orb }}=0.42$ (Buckley et al. 1998) and 0.68 (Córdova et al. 1985) respectively. If the spin period in OY Car really is close to $2240 \mathrm{~s}$ then it begs the question, is OY Car an Intermediate Polar?

\subsection{The mass of the binary components}

The most likely source of X-rays is from a boundary layer of negligible height which is extended in azimuth around the white dwarf. In Sect. 3.5 we showed that in X-rays the ingress and egress of the eclipse was very rapid taking 20-30 s. In addition, the duration of the eclipse in X-rays was $290 \pm 10 \mathrm{~s}$. Using the observed times, we can place constraints on the system parameters.

There is good agreement regarding the system inclination: $83^{\circ}$ (cf. Wood et al. 1989). There is, however, a wide variation in the quoted mass of the white dwarf, $M_{1}$, varying from $0.33-1.26 M_{\odot}$. The mass of the secondary star, $M_{2}$, is not much better constrained, with estimates ranging from $0.07-0.15 M_{\odot}$. There is better agreement on the mass ratio, $q=\left(M_{2} / M_{1}\right)$, where the more recent estimates quoted in Wood et al. (1989) gave $q=0.10-0.11$.

Using an inclination $i=83.3^{\circ}$ (Wood et al. 1989) we determined the ingress time and total eclipse duration for a range of $M_{1}, M_{2}$. We assume the Nauenberg (1972) mass-radius relationship for a white dwarf and the secondary star is a main sequence star (i.e. it is not overmassive for its spectral type as has been suggested for some secondary stars).

We determine the size of the eclipsed source by tracing the Roche potential out of the binary system along the line of sight from any point in the vicinity of the white dwarf. We then determine the ingress duration and eclipse duration for various combinations of $M_{1}, M_{2}$. Comparing these times with the observed times (Sect. 3.5) we find that only for $M_{2}=0.08-0.11 M_{\odot}$ and $M_{1}=0.9-1.1 M_{\odot}$ can we match the observed times. Compared to previous estimates of the component masses these are tightly constrained. If the boundary layer has a non-negligible height, then $M_{1}$ will be more massive.

\section{References}

Aschenbach, B., Briel, U., Haberl, F., et al. 2000, SPIE, 4012, Paper 86 [astro-ph/0007256]

Buckley, D. A., H., Cropper, M., Ramsay, G., \& Wickramasinghe, D. T. 1998, MNRAS, 299, 83

Córdova, F. A., Mason, K. O., \& Kahn, S. M. 1985, MNRAS, 212,447

den Herder, J. W., Brinkman, A. C., Kahn, S. M., et al. 2001, A\&A, 365, L7

Horne, K. Marsh, T. R., Cheng, F. H., Hubeny, I., \& Lanz, T. 1994, ApJ, 426, 294

Jansen, F., Lumb, D., Altieri, B., et al. 2001, A\&A, 365, L1

Malkov, O., Yu., Pishunov, A. E., \& Shpil'kina, D. A. 1997, A\&A, 320, 79

Marsh, T. R., \& Horne, K. 1998, MNRAS, 299, 921

Mason, K. O., Cordova, F., Watson, M. G., \& King, A. R. 1988, MNRAS, 232, 779

Mason, K. O., Breeveld, A., Much, R., et al. 2001, A\&A, 365, L36

Mattei, J. A. 2000a, Observations from the AAVSO online "quick look" data file, private communication

Mattei, J. A. 2000b, Observations from the AAVSO International database, private communication

Mauche, C., Raymond, J. C. 2000, in press ApJ [astro-ph/0004414]

Mukai, K., Wood, J. H., Naylor, T., Schlegel, E. M., \& Swank, J. H. 1997, ApJ, 475, 812

Nauenberg, M. 1972, ApJ, 175, 417

Naylor, T., Bath, G. T., Charles, P. A., et al. 1988, MNRAS, 231,237

Pratt, G. W., Hassall, B. J. M., Naylor, T., \& Wood, J. H. 1999, MNRAS, 307, 413

Ramsay, G., Córdova, F., Cottam, J., et al. 2001, A\&A, 365, L294

Randich, S., Schmitt, J. H. M. M., Prosser, C. F., \& Stauffer, J. R. 1996, A\&A, 305, 785

Steiman-Cameron, T. Y., \& Imamura, J. N. 1999, ApJ, 515, 404

Turner, M. J. L., Abbey, A., Arnaud, M., et al. 2001, A\&A, $365, \mathrm{~L} 27$

van Tesseling, A. 1997, A\&A, 319, L25

Wood, J. H., Horne, K., Berriman, G., \& Wade, R. A. 1989, ApJ, 341, 974

Wood, J. H., Naylor, T., Hassall, B. J. M., \& Ramseyer, T. F. 1995, MNRAS, 273, 772 